Artigo Original

Original Article

Suelly Cecilia Olivan Limongi

Emilia de Faria Oliveira ${ }^{1}$

Livia Maria lenne ${ }^{1}$

Rosangela Viana Andrade ${ }^{1}$

Angela Maria de Amorim Carvalho ${ }^{2}$

Descritores

Síndrome de Down Desenvolvimento da linguagem Desenvolvimento infantil Criança

Linguística

Estudos de linguagem Transtornos da linguagem

Keywords

Down syndrome

Language development Child development

Child

Linguistics

Language arts Language disorders

Endereço para correspondência: Suelly Cecilia Olivan Limongi

R. Cipotânea, 51, Cidade Universitária, São Paulo (SP), Brasil, CEP: 05360-160.

E-mail: slimongi@usp.br

Recebido em: 10/09/2012

Aceito em: 20/12/2012

\section{Utilização de substantivos e verbos por crianças com síndrome de Down em duas situações diferentes}

\author{
The use of nouns and verbs by children with Down \\ syndrome in two different situations
}

\section{RESUMO}

Objetivo: Verificar a utilização de substantivos e verbos por crianças com síndrome de Down e comparar esse uso em situações de interação com o terapeuta e com a mãe. Métodos: Participaram 21 crianças com idades entre 5 e 11 anos, divididas em 3 grupos igualitários com base nas idades cronológica e mental, a partir da aplicação do Primary Test of Nonverbal Intelligence. A coleta de fala foi feita a partir de situações de interação livre, gravadas em vídeo por período de 30 minutos e transcritas em protocolo específico, sendo utilizados os primeiros 100 enunciados, a partir dos 5 minutos iniciais. O tempo de intervalo entre cada situação foi de 7 a 15 dias. A análise estatística utilizou o teste-t pareado e a análise de variância (ANOVA). Foi adotado o valor de significância de 5\%. Resultados: Foram produzidos mais verbos do que substantivos nas duas situações de interação, sendo mais verbos e substantivos com o terapeuta do que com a mãe. A comparação entre os grupos na interação com o terapeuta mostrou diferença tanto para substantivos quanto para verbos e, com a mãe, apenas tendência à diferença para os verbos. Conclusão: Os dados apontam para crescente desenvolvimento no uso de substantivos e de verbos de acordo com o aumento da idade. Houve maior utilização de verbos que de substantivos, principalmente na situação de interação com o terapeuta.

\begin{abstract}
Purpose: To verify the use of nouns and verbs by children with Down syndrome and to compare this use between conditions of interaction with the mother and the Speech-language pathologist (SLP). Methods: Participants were 21 children aged between 5 and 11 years, divided into three groups, according to chronological and mental age as established by the results of the Primary Tests of Nonverbal Intelligence. The speech sample was obtained through free interaction situations that were videotaped during session of 30 minutes and transcribed in specific protocols. The first 100 utterances from the first five minutes were used. The interval between each situation ranged from 7 to 15 days. $T$-test and analysis of variance were used for statistical analysis and the significance level adopted was of 5\%. Results: More verbs than nouns were used in both conditions; however, a higher number of nouns were observed during the interaction with the SLP. The between-group comparison in the interaction with the SLP shows significant differences for verbs and nouns, but during the interaction with the mother, there was tendency for difference only for the verbs. Conclusion: The data indicate the growing development on using of nouns and verbs according to the increase of age. There was a higher use of verbs when compared with nouns mainly in the condition of interaction with the SLP.
\end{abstract}

Trabalho realizado no Laboratório de Investigação Fonoaudiológica em Síndromes e Alterações SensórioMotoras, Departamento de Fisioterapia, Fonoaudiologia e Terapia Ocupacional, Faculdade de Medicina, Universidade de São Paulo - USP - São Paulo (SP), Brasil.

(1) Curso de Fonoaudiologia, Departamento de Fisioterapia, Fonoaudiologia e Terapia Ocupacional, Faculdade de Medicina, Universidade de São Paulo - USP - São Paulo (SP), Brasil.

(2) Programa de Pós-graduação (Mestrado) em Ciências da Reabilitação, Departamento de Fisioterapia, Fonoaudiologia e Terapia Ocupacional, Faculdade de Medicina, Universidade de São Paulo - USP - São Paulo (SP), Brasil.

Clinical Trials registration number NCT00952354.

Conflito de interesse: nada a declarar. 


\section{INTRODUÇÃO}

Pesquisas que tratam do desenvolvimento de linguagem reforçam a existência de sincronicidade entre o desenvolvimento lexical e o gramatical e apontam que, no início, o desenvolvimento lexical é mais rápido, uma vez que há necessidade de um número mínimo de palavras para que a gramática se desenvolva, inclusive para que permita o uso de preposições e conjunções ${ }^{(1-5)}$. Uma forte fonte de informação para a criança categorizar substantivos e verbos é a co-ocorrência lexical em estruturas frasais semelhantes, isto é, o uso de um mesmo substantivo ou verbo em frases que mantêm a mesma estrutura sintática, o que levará à habilidade em generalizar ${ }^{(1)}$. Outra fonte importante é a posição em que o vocábulo se apresenta na sentença ${ }^{(6,7)}$.

A literatura aponta a presença de maior número de substantivos do que verbos no período inicial do desenvolvimento de linguagem $^{(8,9)}$ e assegura que a diferença entre esses termos diminui com o aumento da idade das crianças ${ }^{(1-3,8)}$. São citados fatores que influenciam na preferência, no período inicial de aquisição, de substantivos sobre verbos ${ }^{(8)}$ : a frequência, a saliência pela posição na sentença, a transparência morfológica e a saliência pragmática.

Em geral, considera-se que substantivos são aprendidos mais cedo e mais facilmente que verbos porque estão relacionados a objetos concretos e básicos, de convivência usual, além de serem conceitualmente mais simples; têm a possibilidade de ser ordenados hierarquicamente em uma categoria; apresentam mais relações semânticas previsíveis que outras classes de palavras ${ }^{(4,7,8)}$.

Para os verbos, argumenta-se que seu surgimento é mais tardio devido a fatores como: estarem diretamente relacionados com o argumento que precedem ou seguem na estrutura frasal; referirem-se a eventos transientes que não são percebidos pela criança pequena, embora aspectos situacionais sejam relevantes para o significado; terem maior probabilidade de ter o significado alterado de acordo com o contexto ${ }^{(8,9)}$.

$\mathrm{Na}$ aquisição e desenvolvimento lexical e na utilização do léxico, muitos autores apontam a grande influência do contexto ambiental e da relação com a mãe. É apontada, também, a forte associação entre a forma como a mãe responde à comunicação de seu filho e o nível de comunicação funcional apresentado pela criança ${ }^{(10)}$, o que incluiria o uso preferencial de substantivos e verbos por mães, que depende de características culturais e da estrutura da língua ${ }^{(11)}$. Cita-se, ainda, o fato de o modo com o qual a criança usa as palavras estar fortemente ligado à frequência e ao padrão de uso dessas palavras por sua mãe $e^{(7)}$, além de dar ênfase à palavra no final da sentença ${ }^{(6)}$.

$\mathrm{Na}$ literatura nacional, são encontrados estudos ${ }^{(12-14)}$ nos quais a evolução no uso do verbo no Português Brasileiro em crianças com desenvolvimento típico mostrou-se compatível com os achados em estudos internacionais, como os citados, e indicaram que a aquisição dessa classe de palavra é baseada no uso e na atenção às pistas que são fornecidas pelo contexto, pelas estruturas sintáticas e semânticas. Por outro lado, os dados dessas pesquisas nacionais mostram que, ao contrário da literatura internacional, a população estudada, cujos participantes tinham entre dois e quatro anos de idade, apresentavam mais verbos que substantivos. Os autores fizeram importante observação no sentido de que tais crianças, por já estarem com mais de dois anos de idade, estavam em fase de expansão no uso de verbos, uma vez que os substantivos já haviam apresentado crescimento importante e que os verbos se faziam necessários para que houvesse a combinação de palavras.

Inúmeros estudos voltados para o desenvolvimento da linguagem da criança com síndrome de Down (SD) ${ }^{(15-20)}$, além de apontarem a importante influência do desenvolvimento cognitivo, como também do meio e da família nesse processo, ressaltam a defasagem existente com relação à compreensão e expressão oral, sendo esta última mais afetada. Quanto ao vocabulário, para alguns autores ${ }^{(15,17,20,21)}$, o que concorre para a eficiência na aquisição lexical e, posteriormente, combinação de palavras em sentenças na criança com SD, é a frequência com a qual as palavras são usadas e a posição que ocupam dentro da frase, principalmente com referência a substantivos e verbos.

Ao ser estudado o papel da mãe como participante ativa nesse processo de aprendizagem, autores ${ }^{(22,23)}$ referem que crianças com atraso no desenvolvimento de linguagem, incluindo aquelas com SD, apresentam determinadas características que favorecem a utilização, por parte de seus pais, de frases mais curtas, com sintaxe e morfologia simplificadas. Essas características podem ser sintetizadas em ininteligibilidade, uso de inapropriações gramaticais, dificuldades para iniciar, sustentar e adequar tópicos conversacionais.

Ao se considerar a intervenção terapêutica em linguagem com essas crianças e ao voltar o foco de atenção no papel do terapeuta, estudos discutem, de forma geral, a atuação desses profissionais ${ }^{(24-26)}$, mas poucos são os que se referem à intervenção com crianças com defasagem cognitiva e, mais especificamente, com a SD ${ }^{(10,25)}$.

A partir do exposto, são propostos como objetivos do presente estudo verificar a utilização de substantivos e verbos por crianças com SD e comparar esse uso em situações de interação com o terapeuta e a mãe.

\section{MÉTODOS}

Esta pesquisa foi aprovada pela Comissão de Ética para Análise de Projetos de Pesquisa da instituição, sob n ${ }^{\circ}$ 0940/07, e iniciada somente após a leitura e aprovação, por parte dos responsáveis, do Termo de Consentimento Livre e Esclarecido.

Participaram 21 crianças com SD com idade cronológica entre 5 e 11 anos, divididas em 3 grupos com 7 crianças em cada, de acordo com a idade mental (IM) obtida por meio do Primary Test of Nonverbal Intelligence (PTONI) ${ }^{(27)}$, que fornece esse dado baseado na inteligência não verbal para habilidades cognitivas. Os grupos foram compostos por G1: IM entre 3 anos e 3 anos e 11 meses (média de idade de 3 anos e 1 mês) ; G2: IM entre 4 anos e 4 anos e 11 meses (média de idade de 4 anos e 2 meses); G3: IM entre 5 anos e 5 anos e 11 meses (média de idade de 5 anos e 3 meses).

Como critérios de inclusão foram considerados: cariótipo por trissomia simples do cromossomo 21; IM entre 3 anos e 5 anos e 11 meses; resultados audiológicos sugestivos de audição normal; ausência de deficiência visual, de distúrbios psiquiátricos e/ou psicológicos e de origem neurológica; estarem sob intervenção fonoaudiológica na instituição há pelo menos um ano.

A coleta das amostras de fala foi feita a partir da interação terapeuta-criança e mãe-criança, com utilização de brinquedos 
durante o jogo simbólico, gravada em vídeo por um período de 30 minutos e transcrita em protocolo específico. Com o objetivo de controlar a interferência do tempo entre as duas situações de interação, foi considerado como intervalo o período de 7 a 15 dias. Não foi estabelecida ordem para a realização da situação de interação para cada criança.

A situação de interação terapeuta-criança foi obtida com o terapeuta de cada criança, com o objetivo de controle de possível interferência da pessoa componente da díade com quem as crianças não estivessem familiarizadas no contexto terapêutico. $O$ contato entre a díade deveria estar sendo realizado há pelo menos seis meses. Apesar de as díades serem compostas por diferentes terapeutas, todos estavam sob capacitação pelo mesmo modelo terapêutico e sob supervisão com os mesmos profissionais fonoaudiólogos.

Para a transcrição das amostras de fala, foram utilizados os 100 primeiros segmentos (enunciados), sendo que os cinco primeiros minutos foram descartados por terem sido considerados como período de adaptação da criança a cada situação. Para a divisão dos segmentos, utilizou-se o mesmo critério adotado em outra pesquisa com crianças brasileiras ${ }^{(12)}$, que aborda estudo sobre a extensão média de enunciados (EME). Assim, segmento, ou enunciado, foi considerado como a emissão oral ocorrida até a mudança de assunto por parte da criança, ou até a mudança do foco de atenção da criança, ou ainda até a interrupção do interlocutor, e contabilizados os substantivos e verbos.

Os dados foram submetidos a análise estatística, cujos testes utilizados foram o teste- $t$ pareado e análise de variância (ANOVA). O valor de significância adotado foi o de $p \leq 0,05$.

\section{RESULTADOS}

A Tabela 1 apresenta a estatística descritiva do desempenho das crianças como grupo único para cada categoria gramatical, isto é, substantivos e verbos, em cada um dos contextos de interação.

Tabela 1. Desempenho de todas as crianças para cada categoria gramatical nos contextos de interação

\begin{tabular}{lccc}
\hline Situação & Média \pm DP & Min-Máx & Med. $\left(1^{\circ} \mathrm{Q}-3^{\circ} \mathrm{Q}\right)$ \\
\hline Terapeuta & & & \\
$\quad$ Substantivo & $36,5 \pm 20,5$ & $7,0-90,0$ & $33,0(22,0-47,0)$ \\
$\quad$ Verbo & $70,1 \pm 26,5$ & $22,0-133,0$ & $71,0(51,0-89,5)$ \\
Mãe & & & \\
$\quad$ Substantivo & $43,6 \pm 18,4$ & $17,0-83,0$ & $43,0(31,0-50,5)$ \\
$\quad$ Verbo & $55,5 \pm 25,7$ & $17,0-99,0$ & $55,0(32,0-74,5)$ \\
\hline
\end{tabular}

Legenda: $\mathrm{DP}$ = desvio-padrão; Min = mínimo; Máx = máximo; Med. = mediana; $1^{\circ} \mathrm{Q}=1^{\circ}$ quartil; $3^{\circ} \mathrm{Q}=3^{\circ}$ quartil

Observa-se que as crianças produziram mais verbos do que substantivos tanto nas interações com o terapeuta quanto com a mãe, sendo em maior número com o terapeuta. Com relação ao valor máximo, é interessante notar que foi maior na situação com o terapeuta, tanto para substantivo quanto para verbo. Na comparação entre substantivos e verbos em cada situação, o teste- $t$ indicou que o uso de ambos difere com o terapeuta $\left(\mathrm{t}_{20}=5,485\right.$, $\mathrm{p}<0,001)$ e com a mãe $\left(\mathrm{t}_{20}=2,152, \mathrm{p}=0,044\right)$. Na comparação entre terapeuta e mãe para cada classe gramatical, o teste- $t$ indicou que o uso de substantivos não difere dependendo do interlocutor $\left(\mathrm{t}_{20}=1,465, \mathrm{p}=0,158\right)$; porém, há diferença para o uso de verbo $\left(\mathrm{t}_{20}=2,958, \mathrm{p}=0,008\right)$, com maior ocorrência com o terapeuta.
A Tabela 2 apresenta a estatística descritiva do desempenho de cada grupo para cada categoria gramatical, substantivos e verbos, em cada um dos contextos de interação.

Tabela 2. Desempenho de cada grupo para as categorias gramaticais nos contextos de interação

\begin{tabular}{|c|c|c|c|c|}
\hline & \multicolumn{2}{|c|}{ Terapeuta } & \multicolumn{2}{|c|}{ Mãe } \\
\hline & Substantivo & Verbo & Substantivo & Verbo \\
\hline G1 & $21,6( \pm 10,0)$ & $50,7( \pm 18,7)$ & $35,4( \pm 12,4)$ & $40,1( \pm 23,5)$ \\
\hline $\mathrm{G} 2$ & $37,1( \pm 14,6)$ & $70,0( \pm 19,5)$ & $44,0( \pm 18,6)$ & $53,7( \pm 16,2)$ \\
\hline G3 & $50,9( \pm 24,2)$ & $89,6( \pm 26,8)$ & $51,4( \pm 21,9)$ & $72,6( \pm 27,7)$ \\
\hline
\end{tabular}

${ }^{*}$ Média ( \pm desvio-padrão)

Legenda: G1 = grupo 1; G2 = grupo 2; G3 = grupo 3.

Observa-se que há aumento gradual dos valores correspondentes à média de uso de substantivos e verbos de acordo com o aumento das idades dos grupos, para cada situação de interação.

A Tabela 3 apresenta a comparação entre os grupos, para cada classe gramatical, na situação de interação com o terapeuta.

Tabela 3. Comparação entre os grupos, para substantivos e verbos, na interação com o terapeuta

\begin{tabular}{ccccc}
\hline & $\begin{array}{c}\text { Diferença } \\
\text { média }\end{array}$ & $\begin{array}{c}\text { Erro } \\
\text { padrão }\end{array}$ & Valor de p & IC \\
\hline Substantivo & & & & \\
G1xG2 & $-15,57$ & 9,27 & 0,240 & $-39,2-8,1$ \\
G1xG3 & $-29,29$ & 9,27 & $0,014^{*}$ & $-52,9--5,6$ \\
G2xG3 & $-13,71$ & 9,27 & 0,324 & $-37,4-9,9$ \\
Verbo & & & & \\
G1xG2 & $-19,29$ & 11,76 & 0,255 & $-49,3-10,7$ \\
G1xG3 & $-38,86$ & 11,76 & $0,010^{*}$ & $-68,9-8,8$ \\
G2xG3 & $-19,57$ & 11,76 & 0,246 & $-49,6-10,4$ \\
\hline
\end{tabular}

*valores significativos $(p<0,05)$ - Teste de Tukey; IC = intervalo de confiança Legenda: $\mathrm{G} 1$ = grupo 1; G2 = grupo 2; G3 = grupo 3.

A ANOVA de um fator revelou haver diferença entre os grupos tanto para o substantivo ( $\mathrm{F}=5,001, \mathrm{gl}=2, \mathrm{p}=0,019)$, quanto para o verbo $(\mathrm{F}=5,461, \mathrm{gl}=2, \mathrm{p}=0,014)$. $\mathrm{O}$ teste de Tukey indicou que essa diferença se restringe à comparação entre os grupos 1 e 3, para ambas as classes gramaticais.

A Tabela 4 apresenta a comparação entre os grupos, para cada classe gramatical, na situação de interação com a mãe.

Tabela 4. Comparação entre os grupos, para substantivos e verbos, na interação com a mãe

\begin{tabular}{ccccc}
\hline & $\begin{array}{c}\text { Diferença } \\
\text { média }\end{array}$ & $\begin{array}{c}\text { Erro } \\
\text { padrão }\end{array}$ & Valor de p & IC \\
\hline Substantivo & & & & \\
G1xG2 & $-8,57$ & 9,66 & 0,655 & $-33,2-16,1$ \\
G1xG3 & $-16,00$ & 9,66 & 0,249 & $-40,7-8,7$ \\
G2xG3 & $-7,43$ & 9,66 & 0,726 & $-17,2-32,1$ \\
Verbo & & & & \\
G1xG2 & $-13,57$ & 12,26 & 0,522 & $-44,9-17,7$ \\
G1xG3 & $-32,43$ & 12,26 & $0,042^{*}$ & $-63,7--1,1$ \\
G2xG3 & $-18,86$ & 12,26 & 0,297 & $-50,1-12,4$ \\
\hline
\end{tabular}

*valores significativos $(p<0,05)$ - Teste de Tukey; IC = intervalo de confiança Legenda: $\mathrm{G} 1=$ grupo $1 ; \mathrm{G} 2$ = grupo 2; $\mathrm{G} 3=$ grupo 3 . 
A ANOVA de um fator revelou não haver diferença entre os grupos para os substantivos $(\mathrm{F}=1,373, \mathrm{gl}=2, \mathrm{p}=0,279)$, mas indicou tendência à diferença para o verbo $(\mathrm{F}=3,528, \mathrm{gl}=2$, $\mathrm{p}=0,051)$. $\mathrm{O}$ teste de Tukey confirmou não haver diferença entre os grupos para os substantivos, mas confirmou diferença para os verbos entre os grupos 1 e 3 .

\section{DISCUSSÃO}

No presente estudo, os participantes foram agrupados por desenvolvimento cognitivo, por meio de teste que fornece esses dados baseados na inteligência não verbal para habilidades $\operatorname{cognitivas}^{(27)}$, com a finalidade de minimização da variabilidade entre idade cronológica e desenvolvimento $\operatorname{cognitivo~}^{(28)}$. São muitos os estudos que remetem à variabilidade de desempenho nas diferentes áreas que compõem o desenvolvimento de crianças com SD, como cognitiva, linguagem, social, emocional e motora. Alguns estudos apresentam achados em relação à linguagem quanto ao desenvolvimento e alterações encontradas ${ }^{(15-21)}$. Sugere-se, assim, que os participantes de estudos sejam agrupados por idade mental, além de, algumas vezes, tal organização ser aliada à idade cronológica ${ }^{(16,17,19,21)}$.

Outro cuidado tomado foi acerca da participação dos interlocutores formadores das díades que seriam observadas nas duas situações de interação. Optou-se pela participação de mães, uma vez que a literatura, na maioria das vezes, responsável por esse tipo de estudo, apresenta os dados obtidos com este interlocutor ${ }^{(10,22,23,29)}$. Com relação ao terapeuta, a opção feita foi de manutenção do profissional que já se encontrava em trabalho de intervenção terapêutica com a criança participante, com o objetivo de ser evitada interferência da não familiaridade entre as díades. Para garantir a homogeneidade no método de trabalho terapêutico, todos os terapeutas estavam sob a mesma orientação há pelo menos nove meses, como sugerem estudos acerca da formação do profissional que trabalha com linguagem ${ }^{(10,24-26)}$.

Os achados referentes à análise dos dados obtidos a partir das respostas de todos os participantes do presente estudo revelaram que as crianças utilizaram mais verbos que substantivos nas duas situações consideradas, isto é, na relação com a mãe e com o terapeuta, diferentemente do encontrado na literatura, quando se refere ao período inicial do desenvolvimento de linguagem ${ }^{(4,5,8,9)}$. Alguns estudos apontam o caráter de estruturação sintática relacionado ao verbo, com dependência à estrutura da língua. Nesse sentido, o Português tem no uso do verbo fator relacionado a tal estruturação ${ }^{(8,12)}$. Estudos nacionais voltados à utilização de classes de palavras por crianças em fase de desenvolvimento de linguagem apontam esse fator como uma das justificativas para a maior utilização de verbos nas populações estudadas ${ }^{(12,13)}$. Os dados da presente pesquisa corroboram esses resultados.

A literatura aponta que a aprendizagem de substantivos ocorre durante o primeiro ano de vida da criança, intensifica-se durante o segundo ano e são facilmente aprendidos devido a fatores como a frequência no uso pelo adulto, sua relação com objetos concretos e sua simplicidade conceitual, além da saliência pragmática ${ }^{(4,5,7,8)}$. A maior utilização de verbos segue esse período, uma vez que sua função é favorecer a estruturação sintática dos enunciados, fundamental para a adequada combinação de palavras ${ }^{(3,6-8)}$. Tais fatores, em síntese, justificariam a predominância de substantivos sobre verbos nos períodos iniciais do desenvolvimento de linguagem ${ }^{(4,5,7-9)}$, mas equiparado com o aumento da idade das crianças ${ }^{(3,6,8)}$. Estudos comparativos entre línguas de diferentes raízes apontam que as de origem românica, entre elas o Português, possuem maior riqueza morfológica em relação a verbos, com flexões relacionadas a tempo, modo, número e pessoa, o que dificultaria a aquisição pela criança nova ${ }^{(2,6,8,12,13,28)}$.

Outros fatores que favorecem os achados sobre maior utilização de verbos pelos participantes deste estudo fazem referência ao processo de aprendizagem de verbos novos, no período inicial de aprendizagem da linguagem, em que a criança usará pistas sintáticas para interpretar sentenças com esses verbos ${ }^{(1)}$ e contará com a ajuda de verbos mais gerais (utilizados no contexto com maior frequência), importantes porque permitirão descobrir padrões sintáticos que regem grupos de verbos ${ }^{(4)}$. É mencionada, ainda, a influência do domínio semântico de um significado particular para a criança, como a utilização de ações de solicitação ou de atenção, de localização e de indicação, que podem estar envolvidas em determinados verbos ou na maneira como são utilizados ${ }^{(8)}$, o que ocorre com frequência na comunicação do adulto em relação à criança.

Os achados da presente pesquisa corroboram o que é apontado a respeito da influência da idade das crianças estudadas. Pesquisas que têm como foco o desenvolvimento de linguagem típico apontam que a preferência pelo uso de substantivos a verbos se dá no período inicial da aquisição lexical, até por volta dos dois anos de idade, quando se iniciará um aumento linear pelo uso de verbos ${ }^{(3,7,8)}$. Essa questão foi mencionada nos estudos nacionais ${ }^{(12,13)}$, ao ser comentado que tais participantes tinham entre dois e quatro anos de idade, o que pode ter interferido na obtenção de dados que acusaram maior utilização de verbos que de substantivos, exatamente por se encontrarem em fase de expansão no uso de verbos.

Não se pode desconsiderar que as crianças do presente estudo, embora tivessem desenvolvimento cognitivo que as localizasse entre 3 e 5 anos de idade, cronologicamente tinham entre 5 e 11 anos, o que lhes renderia benefícios em termos de anos de experiência e de exposição à estrutura de sua língua, apesar da defasagem cognitiva que permeia a SD. A literatura internacional reforça tais comentários e assume a realização de estudos sobre o desenvolvimento linguístico de crianças com SD a partir de, aproximadamente, 24 ou 30 meses de idade, devido ao fato de essas crianças apresentarem tardiamente a expressão oral ${ }^{(16-18,20)}$.

É importante assinalar que, ao serem comparados os desempenhos entre os grupos nas duas situações, houve aumento gradual no uso tanto de verbos quanto de substantivos, mas sempre com predomínio dos verbos. Esse fato demonstra que os participantes encontravam-se em situação de desenvolvimento lexical e gramatical, caminhando para maior qualidade em relação à gramaticalização. De acordo com estudos ${ }^{(2,3,5,6)}$, tal fato permitirá a ocorrência da complexidade gramatical, com o uso de adjetivos, preposições e conjunções. Concorre para esse desenvolvimento o input linguístico do meio, a capacidade linguística da criança e o desenvolvimento cognitivo $^{(3)}$. Novamente, os dados obtidos com as crianças com SD estudadas encontram eco na literatura. 
Estudos que tratam do desenvolvimento lexical, no caso substantivos e verbos, fazem seu relato acerca de situações de observação naturalística, ou mais estruturada, ou ainda por meio de dados obtidos com a aplicação de questionários, e, em geral, contam com a participação de pais, em sua maioria mães ${ }^{(2,6,7,11,23)}$. Autores que estudam patologias, tomando-se como foco a SD, utilizam essas mesmas formas de obtenção de dados e as realizam tanto com mães quanto com terapeutas da área da fala e linguagem ${ }^{(10,22,23,28,29)}$. Considera-se que o parceiro comunicativo e a tarefa sejam componentes importantes do contexto do qual a criança fará parte, e seu desempenho comunicativo pode variar, muito embora não sejam encontradas pesquisas que comparem situações diferentes no estudo do desenvolvimento lexical ${ }^{(22)}$. Dessa forma, a proposta realizada pela presente pesquisa objetivou obter dados que oferecessem a possibilidade de tal comparação. Nesse sentido, os achados mostraram que, em ambas as situações, as crianças com SD utilizaram mais verbos que nomes, como comentado anteriormente. Mas um fato merece atenção: a utilização dessas classes de palavras na situação de interação com o terapeuta foi maior que com a mãe.

O fato de os participantes do presente estudo terem apresentado mais verbos que nomes durante a interação com suas mães pode corroborar achados da literatura que apontam que mães de crianças com SD são mais diretivas em sua comunicação oral, formulam poucas perguntas e utilizam mais sentenças afirmativas, além de fazerem poucas referências ao ambiente. É observado que essas mães referem-se com maior frequência às ações das crianças ${ }^{(22,23)}$. Leva-se em consideração, também, o que é apontado em estudos sobre a importância da posição do vocábulo na sentença, de forma que as mães dão ênfase à palavra final na estrutura frasal e que o modo como a criança usa as palavras está fortemente ligado à frequência e padrão de uso dessas palavras pela mãe $e^{(6,7,10,22)}$.

Autores salientam, também, que pode ser observada forte associação entre a forma como as mães respondem à expressão oral utilizada por seus filhos e o nível de comunicação funcional que as crianças usam, de forma a fazerem ajustes da linguagem oral de acordo com a percepção que têm sobre a competência linguística da criança, sem considerar a idade cronológica $^{(10,22)}$. Embora não tenha sido o foco deste estudo, a análise das situações de interação mãe-criança aponta para a possibilidade de se considerar esses fatos como factíveis para a população estudada, o que sugere a importância de serem pesquisados de forma sistematizada.

A literatura mostra-se incipiente especificamente com relação ao papel do terapeuta de linguagem em situações de interação com crianças com SD com o objetivo de estudo da utilização lexical. É apontado que esses profissionais atuam de forma diretiva, com o objetivo de modelar e eliciar comportamentos comunicativos, entre eles a expressão oral ${ }^{(10)}$. Em pesquisa que teve por objetivo estudar o papel desse profissional em seu trabalho com crianças com defasagem cognitiva de origem não específica, é relatado que o terapeuta busca, também, a adequação do ambiente de comunicação oral com essas crianças ${ }^{(30)}$. Tais fatos não estão distanciados de estudos que, do ponto de vista geral, trazem como foco a atuação do terapeuta de linguagem, em que são apontadas atuações com vistas à preocupação com a realização de tarefas/procedimentos e utilização de materiais, embora, nem sempre, visem à busca de situações realistas em terapia ${ }^{(24,26)}$.

A análise dos dados encontrados sobre a utilização de substantivos e verbos por crianças com SD em interação com mães e com terapeutas permite observar que o papel desenvolvido pelo terapeuta apresentou ações seletivas e maior preocupação com situações que permitissem obter respostas orais por parte dos participantes, o que corrobora a literatura. Chama a atenção, nesse sentido, o maior distanciamento na média do uso entre verbos e substantivos na situação de interação com o terapeuta do que com a mãe.

Estudo realizado com crianças que tinham atraso de linguagem de diferentes etiologias, entre elas crianças com déficit cognitivo, apontou que, durante a intervenção terapêutica, a maior atenção do terapeuta estava voltada para atividades que favorecessem a linguagem oral das crianças, como situações de jogo competitivo e de jogo simbólico, nas quais ficava aparente sua atuação diretiva ${ }^{(30)}$. Durante a terapia fonoaudiológica com vistas ao trabalho de linguagem de crianças com SD em nossa realidade, frequentemente tal situação pode ser observada, muito embora não se tenha, na literatura nacional, trabalhos que tratem especificamente desse fato. A análise dos achados do presente estudo aponta a importância de tal investigação.

Em contrapartida, pode-se observar maior equilíbrio, por parte das crianças, na utilização entre as classes de palavras estudadas durante a interação com as mães. A justificativa para tal fato, embora também mereça estudo mais detalhado, pode estar nos achados apontados na literatura ${ }^{(10,22,23)}$, em que é relatado que mães de crianças com SD adaptam sua linguagem ao nível de desenvolvimento da criança, dirigindo-se a elas com sua atenção voltada à competência e não à idade cronológica de seus filhos, além de buscarem uma comunicação mais funcional e não ideal.

O foco deste estudo foi verificar a utilização de substantivos e verbos por crianças com SD em situações de interação com suas mães e com seus terapeutas. A opção por comparar ambas as situações pautou-se no fato de ser comentada na literatura, com ênfase em um estudo ${ }^{(22)}$, a influência do parceiro comunicativo e do contexto no qual a comunicação é efetuada, muito embora não sejam encontrados estudos comparativos. Uma limitação deste estudo foi a não realização de análise do tipo de estruturas frasais utilizadas por mães e terapeutas, de forma a favorecer análise mais detalhada da relação entre o uso de substantivos e verbos de acordo com o contexto de comunicação. Ressalta-se que, embora não tivesse feito parte do objetivo central do trabalho, a realização de tal estudo merece atenção, como comentado anteriormente.

Por outro lado, a análise geral dos dados encontrados indica um ponto que merece ser considerado. Diz respeito à utilização de alguns dos achados de literatura que podem trazer influência na efetividade do processo terapêutico em linguagem de crianças com SD, com o foco no desenvolvimento lexical e gramatical: posição do vocábulo na sentença ${ }^{(5-7,17)}$; redundância e regularidade da língua ${ }^{(5,11,17)}$; co-ocorrência do vocábulo em estruturas frasais semelhantes ${ }^{(5)}$; imitação do modelo dado pelo adulto e atenção focal na interação aliada a discurso com informações (responsividade por parte do adulto) ${ }^{(6,10,11,15,23,30)}$; referências ao meio e contexto de vivência (situação naturalística), além da utilização de perguntas não diretivas, mas com espera por respostas ${ }^{(23,30)}$. 


\section{CONCLUSÃO}

Os dados obtidos apontam para maior utilização de verbos que de substantivos nas duas situações de interação para as crianças com SD estudadas e com desenvolvimento cognitivo entre três e cinco anos. Ressalta-se que houve maior uso de ambos os tipos de palavras na interação com o terapeuta que com a mãe e, neste último caso, observou-se maior equilíbrio entre tal utilização. Considera-se, também, o aumento no uso tanto de substantivos quanto de verbos de acordo com o aumento da idade dos grupos estudados. A literatura respalda os resultados relacionando-os com a influência que a criança em desenvolvimento de linguagem sofre quanto à estrutura da língua, ao contexto e ao papel de mães e terapeutas nas interações.

\section{AGRADECIMENTOS}

Agradecemos à Fundação de Amparo à Pesquisa do Estado de São Paulo (FAPESP) pelo apoio concedido para a realização desta pesquisa, sob processo $n^{\circ}$ 2010/09239-8.

*EFO, LMI, RVA e AMAC foram responsáveis pela coleta, análise e discussão dos dados; SCOL colaborou com análise e discussão dos dados e orientação geral.

\section{REFERÊNCIAS}

1. Gertner Y, Fisher C, Eisengart J. Learning words and rules: abstract knowledge of word order in early sentence comprehension. Psychol Sci. 2006;17(8):684-91.

2. Dixon JA, Marchman VA. Grammar and the lexicon: developmental ordering in language acquisition. Child Dev. 2007;78(1):190-212.

3. Le Normand MT, Parisse C, Cohen H. Lexical diversity and productivity in French preschoolers: developmental, gender and sociocultural factors. Clin Linguist Phonet. 2008;22(1):47-58.

4. Childers JB. Early verb learners: creative or not? Monogr Soc Res Child Dev. 2009;74(2):133-9.

5. Lany J, Saffran JR. From statistics to meaning: infant's acquisition of lexical categories. Psych Sci. 2010;21(2):284-91.

6. Bornstein MH, Cote LR, Maital S, Painter K, Park SY, Pascual L, et al. Cross-linguistic analysis of vocabulary in young children: Spanish, Dutch, French, Hebrew, Italian, Korean, and American English. Child Dev. 2004;75(4):1115-39.

7. Goodman JC, Dale PS, Li P. Does frequency count? Parental input and the acquisition of vocabulary. J Child Lang. 2008;35(3):515-31.

8. Bassano D. Early development of nouns and verbs in French: exploring the interface between lexicon and grammar. J Child Lang. 2000;27(3):521-59.

9. Spanoudis GC, Natsopoulos D. Memory functioning and mental verbs acquisition in children with specific language impairment. Res Dev Disabil. 2011;32(6):2916-26.

10. Mahoney G, Perales F, Wiggers B, Herman B. Responsive teaching: early intervention for children with Down syndrome and others disabilities. Down Syndr Res Pract. 2006;11(1):18-28.
11. Hoff E. How social contexts support and shape language development. Dev Rev. 2006;26(1):55-88.

12. Araujo K. Aspectos do desenvolvimento gramatical de crianças pré-escolares em desenvolvimento normal de linguagem [dissertação]. São Paulo: Faculdade de Filosofia, Letras e Ciências Humanas, Universidade de São Paulo; 2003.

13. Befi-Lopes DM, Cáceres AM. Aquisição de verbos em pré-escolares falantes do português brasileiro. Rev CEFAC. 2007;9(4):444-52.

14. Befi-Lopes DM, Cáceres AM. Análise da diversidade de verbos enunciados na fala espontânea de pré-escolares brasileiros. Pró-Fono $\mathrm{R}$ Atual Cient. 2010;22(1): 3-6.

15. Bird EKR, Gaskell A, Babineau MD, Macdonald S. Novel word acquisition in children with Down syndrome: does modality makes a difference? J Commun Disord. 2000;33(3):241-66.

16. Ypsilanti A, Grouios G, Alevriadou A, Tsapniki K. Expressive and receptive vocabulary in children with Williams and Down syndromes. J Intellect Disabil Res. 2005;49(Pt 5):353-64.

17. Abbeduto L, Warren SF, Conners FA. Language development in Down syndrome: from the prelinguistic period to the acquisition of literacy. Ment Retard Dev Disabil Res Rev. 2007;13(3):247-61.

18. Roberts J, Price J, Barnes B, Nelson L, Burchinal M, Hennon EA, et al. Receptive vocabulary, expressive vocabulary, and speech production of boys with Fragile X syndrome in comparison to boys with Down syndrome. Am J Ment Retard. 2007;112(3):177-93.

19. van Balkom H, Verhoeven L, van Weerdenburg M. Conversational behaviour of children with Developmental Language Delay and their caretakers. Int J Lang Commun Disord. 2010;45(3):295-319.

20. Martin GE, Klusek J, Estigarribia B, Roberts JE. Language characteristics of individuals with Down syndrome. Top Lang Disord. 2009;29(2):112-32.

21. Vicari S, Caselli MC, Gagliardi C, Tonucci F, Volterra V. Language acquisition in special populations: a comparison between Down and Williams syndromes. Neuropsychologia. 2002;40(13):2461-70.

22. Pino O. The effect of context on mother's interaction style with Down's syndrome and typically developing children. Res Dev Disabil. 2000;21(5):329-46.

23. Venuti P, de Falco S, Esposito G, Zaninelli M, Bonrstein MH. Maternal functional speech to children: a comparison of autism spectrum disorder, Down syndrome, and typical development. Res Dev Disabil. 2012;33(2):506-17.

24. Horton S, Byng S, Bunning K, Pring T. Teaching and learning speech and language therapy skills: the effectiveness of classroom as clinic in speech and language therapy student education. Int J Lang Comm Dis. 2004;39(3):365-90.

25. Law J. The implications of different approaches to evaluating intervention: evidence from the study of language delay/disorder. Folia Phoniatr Logop. 2004;56(4):199-219.

26. Werner S, Grayzman A. Factors influencing the intention of students to work with individuals with intellectual disabilities. Res Dev Disabil. 2011;32(6):2502-10.

27. Ehrler DJ, McGhee RL. Primary Test of Nonverbal Intelligence - PTONI. Texas: Pro-Ed; 2008.

28. Marques SF, Limongi SCO. A extensão média do enunciado (EME) como medida do desenvolvimento de linguagem de crianças com síndrome de Down. J Soc Bras Fonoaudiol. 2011;23(2):152-7.

29. Ruser TF, Arin D, Dowd M, Putnam S, Winklosky B, Rosen-Sheidley $\mathrm{B}$, et al. Communicative competence in parents of children with autism and parents of children with specific language impairment. J Autism Dev Disord. 2007;37(7):1323-36.

30. Marshall J, Goldbart J, Phillips J. Parents' and speech and language therapists' explanatory models of language development, language delay and intervention. Int J Lang Commun Disord. 2007;42(5):533-55. 